\title{
Using Semantic Information for Processing Negation and Disjunction in Logic Programs \\ by
}

\author{
Terry Gaasterland ${ }^{1}$ and Jorge Lobo ${ }^{2}$
}

\begin{abstract}
There are many applications in which integrity constraints can play an important role. An example is the semantic query optimization method developed by Chakravarthy, Grant, and Minker for definite deductive databases. They use integrity constraints during query processing to prevent the exploration of search space that is bound to fail. In this paper, we generalize the semantic query optimization method to apply to negated atoms. The generalized method is referred to as semantic compilation. We show that semantic compilation provides an alternative search space for negative query literals. We also how semantic compilation can be used to transform a disjunctive database with or without functions and denial constraints without negation into a new disjunctive database that complies with the integrity constraints.
\end{abstract}

\section{Introduction}

There are many applications in which integrity constraints can play an important role. For example, before expanding a database query, it is possible to incorporate the integrity constraints into the query to obtain a semantically equivalent query. The new query contains constraint information that prevents the exploration of search space that is bound to fail. In [C(iM90], a semantic query optimization method is described that compiles integrity constraints into queries based on common positive literals that occur in both the constraini and the query. Database rules are optimized at compile time in order to minimize the amount of computation that must be done at run time. If queries are known at compile time, the entire query can be optimized. Otherwise, any remaining optimization is done at run time. Based on the same principles, integrity constraints can be also used to generate cooperative and informative answers and model user needs (see [MC;90, Ciaa.92]).

We generalize the semantic query optimization method to apply to negated atoms. The generalized method is referred to as semantic compilation. This exploration has led to two

\footnotetext{
'Mathematics and (Oomputer Sicience Division. Argonne National Laboratory, Argonne, IL 604.39. EMAIL: gassterland cumcs.anl.gov

${ }^{2}$ Department of Electrical Engineering and ('omputer Science, University of lllinois at ('hicago, Chicago, IL 60680. E.MAIL: jorge(wrecs.uic.edu

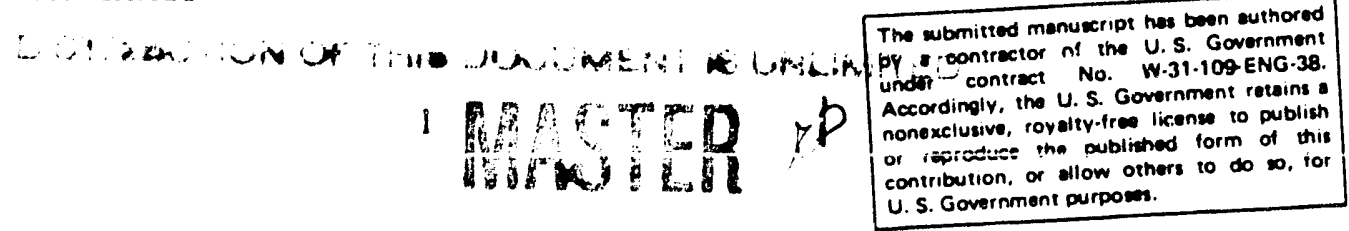


significant results. The first result affords a new method to process negative query literals. Semantic compilation provides an alternative search space for negative query literals. Traversing the alternative search space can find answers in cases for which negation-as-finitefailure and constructive negation may not. To use the negation-as-finite-failure method to expand a negative query literal, the negative literal must be ground, and the search space of the positive ground atom must be finite. To use constructive negation effectively, the literal does not need to be ground but the search space must still be finite. Semantic compilation with negation as described in this paper allows correct answers to be found for negative literals that are neither ground nor finite. However, unless the integrity constraints completely describe all allowed states of the database, the answers for the negative literal may not be complete.

The second result applies to disjunctive deductive databases. Minker and Fernandez [FM91] noticed that there are many situations that can be represented directly with a set of disjunctive statements and a set of constraints that restrict the statements. To answer queries over such databases Fernandez and Minker generate the minimal models of the database and use the constraints to select the models that give the semantics of the database. Their algorithms work for stratified, function-free databases.

We show how for positive disjunctive deductive databases with or without functions and denial constraints without negation, semantic compilation can be adapted to avoid the construction of the minimal models. Other advantages of this approach are that minimal models do not have to be finite, and we can use any proof procedure for disjunctive databases to evaluate queries.

The next section gives background definitions. Section 3 describes and analyzes the method for handling negative literals with semantic compilation. Section 4 describes the compilation of constraints into disjunctive databases.

\section{Background}

Deductive databases are comprised of syntactic information and semantic information. The syntactic information consists of an intensional database (IDB) which is a set clauses (or rules) of the form $A_{1} \vee \cdots \vee A_{m}-B_{1}, \ldots, B_{n}, m, n>0$, where each $A_{i}$ is an atom and each $B_{i}$ is a literal (i.e. an atom or its negation), and the extensional database (EDB) which is the set of clauses of the form $A_{1} \vee \cdots \vee A_{m}-$. IDB clauses are also called rules. $E D B$ clauses are also called facts. If all the $B_{i} s$ in the IDB rules are positive literals and $m=1$, then the deductive database is called definite. If $m$ is larger than 1 in at least one of the clauses, the database is called disjunctive. A pair of literals is said to be a pair of complementary literals if one is an atom and the other a negated atom.

The semantic information about the database consists of a set of integrity constraints (I(:). The constraints considered in this paper have the form $-C_{1}, \ldots, C_{n}^{\prime}, E_{1}, \ldots E_{m}$ where each $C_{i}$ is a literal whose predicate appears in an EDB fact or the head of an IDB rule and each $E_{1}$ is an evaluable expression.

An integrity constraint restricts the states that a database can take. For example, the integrity constraint "No person can be both male and female," - person( $(X)$, male $(X)$, female $(X)$, 
restricts people in a database from having both properties. Because the constraints on a database do not enable the deduction of new answers but rather give information about existing knowledge and answers, they are considered semantic information rather than syntactic information.

Chakravarthy, Grant, and Minker [CGM90] have shown how integrity constraints can be compiled into a query to identify space that is bound to fail. We now describe their algorithm. The algorithm assumes that the EDB and IDB contain function-free definite clauses, and that the IC is a finite set of first order clauses that always hold in the theory EDBUIDB. It is assumed that the set IC contains constraints that can be derived from other constraints in IC.

The semantic optimization process performs two preliminary steps: flattening of the IDB clauses so that all IDB clause bodies contain only EDB predicates or recursive predicates and variable substitution of the IC clauses. Flattening is performed according to [Rei78] and consists of a series of unfoldings of the rules in the IDB. A database with direct recursion can be flattened by flattening the nonrecursive predicates in the recursive rules before merging the rules with the constraints [ $\mathrm{GGL}^{+}+93$ ]. Partial flattening can be done in rules with nondirect recursion by inspecting the dependency graph associated with the rules [ $\mathrm{G}(\mathrm{iL}+93]$. Without loss of generality, but possibly with some loss of potential query processing efficiency at run time, the flattening process does not have to be complete. Moreover, the negative literals are not flattened.

\section{Background definition: Variable substitution}

A variable substituted form of an integrity constraint is a clause obtained by

1. Replacing each distinct constant in the constraint by a distinct variable and adding the fact that the constant equals the variable.

2. Replacing each variable that occurs more than once by new distinct variables. and adding equalities that represent the bindings.

After preprocessing the IDB and the IC using variable substitution, the next step in semantic optimization is to find all partial constraints that apply to each IDB clause. A residue is obtained by determining whether part of some clause in IC partially subsumes an IDB clause. If so, then the remaining part of the IC ciause becomes a residue that constrains the II)B clause - the residue expresses something that must be true when the clause is true. The general form of the integrity constraints that was produced by variable substitution allows us to find all possible residues.

\section{Background definition: Partial subsumption}

A (variable substituted) integrity constraint I partially subsumes a rule $A$ if a subset of I snbbsumes $A$ but $I$ does not subsume $A$. An integrity constraint $I=-C_{1}, \ldots, C_{n}$ partially subsumes a rule $R=A-B_{1}, B_{2}, \ldots, B_{m}$, if and only if there exists a nonempty subset $S$ of $\left\{C_{1} \ldots, C_{n}\right\}$ and a substitution $\theta$ such that $S \theta \subseteq\left\{B_{1}, \ldots, B_{n}\right\}$.

Background definition: Residue

If $C_{i_{1}}, \ldots, C_{i_{k}}$ are the literals in the constraint that are not in $S$, then the elause $-\left(C_{i_{1}}, \ldots, C_{i_{k}}\right) \theta$ is the residue obtained from $R$ and $I$.

Once a set of residues is found for an IDB clause, each residue may be incorporated 
into the clause. Attaching the residue to the IDB clause produces a semantically equivalent clause.

Background definition: Semantical!* constrained rules A semantically constrained rule is defined to be of the form

$$
A-B_{1}, \ldots, B_{m},\left\{R_{1}, \ldots, R_{n}\right\} \text {. }
$$

Here $A$ is the procedure head of the rule, each literal $B_{i}$ is either a regular literal or an evaluable predicate, and the $R_{j}$ s are residues obtained from the rule $A-B_{1}, \ldots, B_{m}$ and the integrity constraints.

\section{Background definition: Semantically constrained database}

Let $D$ be a definite database and let $E, I$, and $C$ be the EDB, IDB, and $I C$ s that form $D$. The semantically constrained database of $D, D^{\prime}$, is the set of semantically constrained rules obtained from $D$.

Consider a database consisting of two IDB clauses:

$$
\text { economic_ties }(C l, C 2)-\operatorname{trade}(C l, Z), \operatorname{trade}(Z, C \Omega) \text {. }
$$

trade(Cl,C2) - friends $(C l, C 2)$.

meaning C.1 has economic ties with C.2 if $C 1$ trades with some $Z$ that trades with $C$ and $C l$ trades with $\left(\%\right.$ if $C l$ is friends with $C^{\prime}$. The variable substituted integrity constraint above partially subsumes the first IDB clause to produce the residue $\{-C l=u s a, C z=i r a q\}$.

A new semantically constrained clause is produced when the residue is merged with the IDB clause

economic_ties(CI,Cs) - trade(CI,Z), trade(Z,C2). $\{-C l=u s a, C a=i r a q\}$.

The constrained axiom says that it is not possible for the countries $\mathrm{Cl}$ and $\mathrm{C} \Omega$ to have economic ties if $\mathrm{Cl}$ is usa and $\mathrm{C} 2$ is irsq.

The semantically constrained database consists of the semantically constrained clauses together with the EDB facts and the integrity constraint above.

\section{Handling Negative Literals}

The semantic optimization method [C(GM90] augments queries and rules with semantic information about the search space of positive atoms in a query or the rules when those atoms occur positively in the body of one or more integrity constraints. In this section, we generalize the approach so that it provides semantic information about the search space of negative as well as positive literals in the query. The generalized method handles all cases in which literals with the same predicate occur positively or negatively in one or more constraints. Semantic information can be obtained in four cases:

1. When a predicate symbol of a positive constraint literal appears in a positive literal in the query or the rules.

2. When a predicate symbol of a negative constraint literal appears in a negative literal in the query or the rules.

3. When a predicate symbol of a negative constraint literal appears in a positive literal in the query or the rules. 
4. When a predicate symbol of a positive constraint literal appears in a negative literal in the query or the rules.

Case 1 is covered in [CGM90] and forms the basis for the treatment of Cases 2, 3 and 4 . Case 2 can be treated like Case 1 by temporarily renaming the negated predicates as unique new positive predicates and applying semantic compilation as in case 1 . Case 3 reduces to using the integrity constraint as a deductive rule and is not particularly interesting. Case 4 is the most interesting: semantic compilation with negation provides a means to find answers for queries that contain possibly nonground negative literals, in which case regular procedures such as SLDNF-resolution do not apply, and/or possibly an infinite number of answers, in which case approaches such as constructive negation [Cha88, Prz89b] cannot be used.

\subsection{Algorithm for Generalized Semantic Compilation}

Semantically compiling a set of integrity constraints with a database rule or query has two main steps: (1) obtaining partial constraints, and (2) integrating the partial constraints into the body of the query or rule. A query may be regarded as a rule of the form Query $-Q_{1}, \ldots, Q_{n}$. From now on, we will refer to both queries and rules as axioms. To compile an integrity constraint into an axiom based on the correspondence of a positive or negative axiom literal and a positive or negative integrity constraint literal, we define mixed partial subsumption. The term mixed refers to the fact that the correlation is done on two complementary literals, one from the constraint and one from the axiom. Since the residues produced by mixed partial subsumption contain a different type of information from the constraints found by regular partial subsumption, we call them mixed residues. We also define a merging algorithm that replaces the axiom literal with the disjunction of its mixed partial constraints.

\section{Definition 1. Mixed partial subsumption}

The mixed partial subsumption of an axiom $R=A-B_{1}, B_{2}, \ldots, B_{m}$, by an integrity constraint $I=-C_{1}, \ldots, C_{n}$, can occur if and only if there exist a $B_{i}$ and a $C_{j}$ such thar.

1. $B_{i}$ and $C_{j}$ are complementary literals.

2. There is a substitution $\theta$ such that $C_{j} \theta \equiv \neg B_{i}$.

The clause $\left(-\left({ }_{1}, \ldots,\left(_{j-1}, C_{j+1}, \ldots, C_{n}\right) \theta\right.\right.$, is the mixed residue obtained from $R$ and I.

The integrity constraint $-\left(C_{1}, \ldots, C_{n}\right.$ can be interpreted to say that it is not the case that $C_{1}$, and $C_{2} \ldots$ and $C_{n}$ are simultaneously true in the database. In particular, if the constraint is restricted with the substitution $\theta$, it is not the case that the conjunction $C_{1} \theta \wedge \cdots \wedge C_{n} \theta$ is true in the database. Hence, if the conjunction $C_{1} \theta \wedge \cdots \wedge C_{j-1} \theta \wedge$ $C_{j+1} \theta \wedge \cdots \wedge C_{n}^{\prime} \theta$ is true, then it is not possible for $C_{j}^{\prime} \theta$ to be true. Since in deductive databases negation is interpreted as negation-as-failure, this is equivalent to saying that $\neg\left({ }_{j}^{\prime} \theta\right.$ is true. In other words, we can rewrite the constraint to be $\left(\neg C_{j}^{\prime} \theta-C_{1} \theta, \ldots, C_{j-1}^{\prime} \theta\right.$, $\left.C_{j+1}^{\prime} \theta, \ldots, C_{n}^{\prime} \theta\right)$. But notice that $\neg C_{j}^{\prime} \theta \equiv B_{i}$. Therefore, we have $\left(B_{i}-C_{1}^{\prime} \theta, \ldots, C_{j-1}^{\prime} \theta\right.$, $\left({ }_{j+1} \theta, \ldots,\left({ }_{n} \theta\right)\right.$. The mixed residue obtained through mixed partial subsumption defines a 
search space that is an alternative to the search space of $B_{i}$. This new implication deduced from the semantic information motivates the following definition of merging a rule with an integrity constraint.

\section{Definition 2. Mixed merging}

To merge the mixed residue $\left(C_{1}, \ldots, C_{j-1}, C_{j+1}, \ldots, C_{n}^{\prime}\right) \theta$ with the rule $R$, replace the rule by the new rule

$$
A-B_{1}, B_{2}, \ldots, B_{i-1}, C_{1} \theta, \ldots, C_{j-1} \theta, C_{j+1} \theta, \ldots, C_{n} \theta, B_{i+1}, \ldots, B_{m} .
$$

Algorithm 1. Semantic Compilation with Negations

Let $D B$ be an initial deductive database. Let $D B^{\prime}$ contain only the $E D B$ of $D B$. Then,

1. For each integrity constraint in the database, use rivixed partial subsumption to find all mixed residues between the integrity constraint and the axiom.

2. For each literal in the axiom that has a corresponding mixed residue, use mixed merging to replace the literal with the mixed residue.

3. For each integrity constraint in the database, use partial subsumption to find residues for the new axiom and the original flattened axiom in $D B$.

4. Add all regular residues to the new axiom and the original axiom using the usual merge definition. Add the new axioms to $D B^{\prime}$.

$D B^{\prime}$ contains the new semantically compiled database.

This algorithm performs semantic compilation for cases 1,3 , and 4 above. The following example illustrates the algorithm.

The database below describes two disjoint groups of points connected by edges (i.e., two distinct connected components of the graph). The disjointness can be described by an integrity constraint. The predicate reachable computes all ordered pairs that are connected by an edge. The predicate unreachable has a search tree with a cycle on reachable. IC:1 is sufficient to describe the disjoint edge groups. The database consists of the following clauses:

$$
\begin{aligned}
& \text { \% group 1: } \quad \text { \% group 2: } \quad \operatorname{reachable}(X, Y)-\operatorname{edge}(X, Y) \text {. } \\
& \text { edge }(a, b) . \quad \operatorname{edge}(c, d) . \quad \operatorname{reachable}(X, Y)-\operatorname{reachable}(X, Z), e d g e(Z, Y) . \quad e d g e(b, a) \text {. } \\
& \text { edge(d.r). unrrachable }(X, Y)-\operatorname{rrachable}(X, Y) \text {. } \\
& \text { I(1: - reachable(a,WI), reachable( } \because W 1) \text {. }
\end{aligned}
$$

Variable substitution of the integrity constraint produces a new form of the constraint: reachuble( $A, W 1)$, reachable $(C, W 2), A=a,(=c, W l=W 2$. Mixed partial subsumption of $I C 1$ with the recursive rule for unreachable produces the following set of residues: $\quad\{-$ reachable(C,X2),X=a,C=c,Y=X2.

- reachabie( $A, X I), X=c, A=a, Y=X I$. . Merging the residues with the rule produces the following new rule:

$$
\begin{aligned}
\text { unreachable }(X, Y)- & ((\neg \text { reachable }(X, Y)) \vee(\operatorname{reachable}(C, X \Omega), X=a,(:=c, Y=X \Omega) \vee \\
& (\operatorname{reachable}(A, X l), A=a, X=c, Y=X(1)) .
\end{aligned}
$$

Unfolding the semantically compiled rule gives three new rules:

$$
\begin{aligned}
& \text { R1: unreachable( } X, Y) \text { - areachable }(X, Y) \text {. } \\
& \text { K2: unrearhable( } X, Y)-\operatorname{reachable(}(X, X), X=a, C=r, Y=X \text { ? } \\
& \text { R:3: unrearhable(X,Y) - rrachable }(A, X /), X=r, A=a, Y=X 1 \text {. }
\end{aligned}
$$

$\mathrm{R} 2 \mathrm{and} \mathrm{R} 3 \mathrm{3}$ can be reduced to the following: 
$\mathrm{R} \cdot 2^{\prime}:$ unreachuble $(a, Y)$ - reachable $(c, Y) . \quad \mathrm{R}: 3^{\prime}:$ unreachable $(c, Y)$ - reachable $(a, Y)$.

From $\mathrm{Rl}$, which contains a negative literal, the query - unreachable $(X, Y)$ cannot be answered using a procedure such as SLDNF-resolution since the unground query — - reachable $(X, Y)$ has to be solved. One possibility is to extend the proof procedure with constructive negation. However, to use constructive negation, one must carefully extend SLDNF-resolution to incorporate constructive answers. Even though there is a finite number of answers for the query — reachable $(X, Y)$, a simple application of SLD-resolution to obtain the answers will result in an infinite computation. However, with $\mathrm{R} 2^{\prime}$ and $\mathrm{R} 3^{\prime}$, produced by Algorithm 1 , two answer substitutions can be computed: $\{X / a, Y / d\}$ and $\{X / a, Y / c\}$. Moreover, suppose the integrity constraint $=-$ reachable $(a, W 1)$, reachable $(c, W 2)$, reachable $(W 1, W 2)$.

is also added to the database. Then, after the compilation, the following two rules are generated:

R4: unreachable $(X, Y)$ - reachable $(a, X)$, reachable $(c, Y)$.

R5: unreachable $(Y, X)$ - reachable $(a, X)$, reachable $(c, Y)$.

From these two rules the rest of the answers for unreachable $(X, Y)$ are obtained.

The following theorem formally establishes the correctness of Algorithm 1.

Theorem 1. Let the tuple $D B=(I D B, E D B, I C)$ be a deductive database. Let $D B^{\prime}=$ $\left(I D B^{\prime}, E D B, I C\right)$ be the database obtained after applying Algorithm 1. Let $Q$ be a query. A substitution $\theta$ is a correct answer substitution for $D B \cup\{Q\}$ if and only if $\theta$ is a correct answer substitution for $D B^{\prime} \cup\{Q\}$.

\subsection{Comparison with Constructive Negation}

When a query contains a negative literal, there are two procedural methods for expanding the search space of the negative literal. Negation-as-finite-failure (NAFF), used in SLDNFresolution, expands the entire search tree for the positive atom in the literal. If the entire tree fails finitely, then the negative atom can be considered to be true. NAFF requires the negative literal to be ground and for the positive atom's search tree to be finite. Otherwise, it can return no answers. ('onstructive negation eliminates the need for the negative literal to be grounded. It expands the search tree of the literal's positive atom to obtain all the answer substitutions for the variables in the atom and constructs an expression formed with equalities and inequalities that states values that the variables in the literal can take or cannot take. Basically, any set of values that make the atom in the literal true are disallowed as substitutions for the negated atom. Constructive negation still has the limitation that the search tree associated with the positive atom must be finite. Another disadvantage is that constructive negation requires all the possible answers for the positive atom before it can return the answer for the negation of the atom. This restriction can make the process very long and extremely complex, especially if a single answer suffices the user posing the query to the clatabase.

As in constructive negation, Algorithm 1 enables a search for values of variables in nonground negative literals. It uses constraint information to identify search space that cannot co-orcur with the query's negated literal. The algorithm produces a new semantically equivalent query that covers the search space and constructs substitutions of allowed values for the variables that appear in the negative literal. Unlike constructive negation, the set of substitutions obtained using the algorithm is not necessarily complete. However, the 
answers that are found are correct. For example, consider the following query, constraint and residue: $Q:-\neg P(X, Y, Z), S(Y)$. IC: $-P(X, Y, Z), R(X, Z)$. Residue: $\{R(X, Z)\} \quad$ Suppose the residue $R(X, Z)$ is expanded as a query and produces the answer substitutions $\theta 1=\{\mathrm{a} / \mathrm{X}, \mathrm{b} / Z\}$ and $\theta \cdot 2=\{a / X, b b / Z\}$. The constraint says that no state of the database will contain both $R(X, Z) \theta I$ and $P(X, Y, Z) \theta l$ or both $R(X, Z) \theta 2$ and $P(X, Y, Z) \theta 2$. Thus, any bindings that are obtained for $Y$ through $S(Y)$ can be concatenated with the $\theta 1$ and $\theta 2$ to produce answer substitutions for the query. Suppose expansion of $S(Y)$ produces two substitutions, $\{c / Y\}$ and $\{c c / Y\}$. Then the final set of answer expressions for the query is the following: $\{a / X, c / Y, b / Z\},\{a a / X, c c / Y$, $b b / Z\},\{a / X, c c / Y, b / Z\},\{a a / X, c / Y, b b / Z\}$, Moreover, in a Prolog-like interpreter, the answers are given one at a time, and if only one answer is required, the computation process can be stopped after the first answer is obtained. Other answers for $R(X, Z)$ may exist.

\section{Disjunctive Databases and Constraints}

When a set of integrity constraints IC is associated with a disjunctive database $P$, the semantics of $(P+I C)$ can be described in two basic ways. We consider the set of models associated with $\mathrm{P}$ alone:

1. If each constraint is true in each model, then the database is considered consistent with IC, and together the models give the meaning of the database. Otherwise, the database is considered inconsistent with IC, and the database has no meaning.

2. If there is some model in which all the constraints are true, then the database is considered to be consistent with IC. The set of models in which all constraints are true describes the meaning of the database. Otherwise, the database is considered inconsistent with $\mathrm{IC}$, and the database has no meaning.

For definite databases, under minimal model semantics, these two cases are equivalent because only one model is associated with a database: its minimal model. However, under minimal model semantics, disjunctive databases may be inconsistent with I $:$ by the first definition yet be consistent by the second definition. This is possible because such databases have multiple minimal models.

The following disjunctive database illustrates this point:

(I)B1: covalent_bond $(A, B) \vee$ double_bond $(A, B)-\operatorname{carbon}(A)$, bonded $(A, B)$.

EDB1: rarbon(iteml). EDB2: hydroxyl(item2). E[)B3: bonded(iteml,item2).

The rule ID)BI says, If some entity $A$ is a carbon atom and it is bonded to some other entity $B$. then the bond is either a covalent bond or a double bond. The facts indicate that iteml is a carbon atom, iteme is a hydroxyl group, and the two items are bonded. The database has two minimal models:

$M_{1}=\{$ carbon(iteml), hydroxyl(item:), bonded(itemL,item:), double_bond(iteml,items)\}

$\boldsymbol{M}_{2}=\{$ carbon(iteml), hydroxyl(item:2), bonded(iteml,item2), covalent_bond(iteml,item:)\}

Now consider a constraint that says, Nothing can be a hydroxyl group and be: double-bonded to something else: Ic:1: - double bond $(A, B)$, hydroxyl(B). The first minimal model violates the constraint with the substitution $\{$ item $1 / A$, item2/B $\}$. Thus, according to the integrity constraint, the first minimal model should be disregarded when answering queries to the database. 
Fernandez and Minker have defined an algorithm that takes a disjunctive database without function symbols, computes its minimal models, and elininates the models that are not consistent with the set of constraints. Answers to queries are computed over these models [FM91]. An alternative way to give answers to queries that are consistent with the integrity constraints is to transform the database into a new database that incorporates the integrity constraint information. If all of the minimal models of the new database are consistent with the constraints, then any answers obtained through any disjunctive query processing mechanism would be consistent with the constraints as well.

We modify the definition of mixed partial subsumption and mixed merging in order to describe a procedure that performs such a transformation on (not necessarily function-free) disjunctive databases and constraints without negation.

\section{Definition 3. Disjunctive subsumption}

The disjunctive subsumption of an axiom $R=A_{1} \vee \cdots \vee A_{k} \leftarrow B_{1}, B_{2}, \ldots, B_{m}$, by an integrity constraint $I=-C_{1}, \ldots, C_{n}$, can occur if and only if there exist a $A_{i}$ and a $C_{j}$ such that they unify with mgu $\theta$. The clause $\left(\leftarrow C_{1}, \ldots, C_{j-1}, C_{j+1}, \ldots, C_{n}\right) \theta$, is the disjunctive residue obtained from $R$ and $I$.

\section{Definition 4. Disjunctive merging}

To merge the disjunctive residue $\left(-C_{1}, \ldots, C_{j-1}, C_{j+1}^{\prime}, \ldots, C_{n}\right) \theta$ with the rule $R$, replace the rule by the new rule $\left(A_{1} \vee \cdots \vee A_{i-1} \vee A_{i+1} \vee \cdots \vee A_{k} \leftarrow B_{1}, \ldots, B_{m}, C_{1} \ldots, C_{j-1}, C_{j+1}\right.$, $\left.\ldots, C_{n}^{\prime}\right) \theta$.

Procedure 1. Semantic Compilation in Disjunctive Databases

Repeat the following steps until no I in IC disjunctive subsumes any rule in $P$ :

1. Select I from $I C ;$ let $I C$ be $I C-\{I\}$.

๖. Let $P^{\prime}$ be $P$.

3. While $P^{\prime} \neq \emptyset$ do

(a) Use disjunctive subsumption to obtain all disjunctive residues between I and the. axioms in $P^{\prime}$.

(b) Use disjunctive merging to generate the new disjunctive rules $N R$.

(c) If the empty clause is in NR report that $P$ is inconsistent with IC and stop.

(d) Remove each rule with an empty head from NR and add it to IC.

(e) Let $P$ be $P \cup N R$.

(f) Let $P^{\prime}$ be NR.

Consider our disjunctive deductive database about a carbon atom and a hydroxyl group. When the procedure is applied to IC'1 and EDB2, the first iteration produces the following intermediate constraint: IC2: - double bond(A, item2). The intermediate constraint is added to the database, and the procedure is applied again to produce a new IDB rule, IDB2, from I( ',2 and II)BI:

IDB2: covalent_bond(A,item:) - $\operatorname{carbon}(A)$, bonded(A,item2).

After all the interactions, the final database produced by the procedure consists of the following rules and facts (note the addition of ID) B:3): 


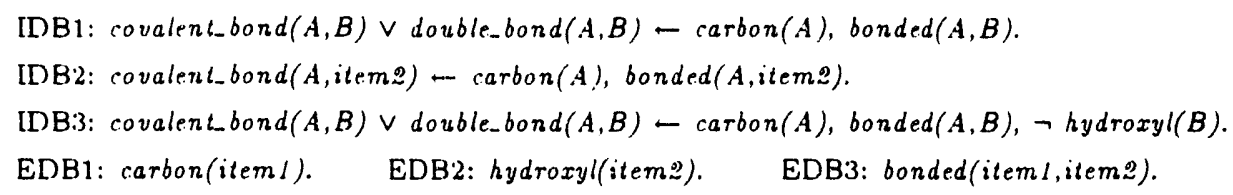

The new database has one minimal model: \{ carbon(iteml), hydroxyl(item2), bonded(item1, item 2), covalent_bond(iteml,item:)\}. The model is consistent with IC1.

Theorem 2. Let $P$ be a positive disjunctive database. Let IC be a set of integrity constraints. Let $P^{\prime}$ be a positive disjunctive database produced by Procedure 1 from $P$ and IC: Then the following holds: (1) Any minimal model of $P$ that satisfies IC is a model for $P^{\prime}$, and (2) Any minimal model of $P$ is a model for $P \cup I C$.

We are not able to guarantee termination of Procedure 1 since it applies to all disjunctive databases and the question of whether or not a set of ICs is consistent with a set of disjunctive clauses is only semi-decidable. However, Procedure 1 is a step toward the extension of semantic compilation to disjunctive theories. We believe that with minor modifications, the same procedure can be used for more general constraints and databases. The modifications will depend on the semantics chosen for negation in disjunctive databases.

\section{Conclusion}

Chakravarthy, Grant, and Minker [CGM90] gave an effective method to optimize queries to definite deductive databases for cases in which partial subsumption involves positive atoms. We have generalized the method to apply to negative literals in either the database axioms or the integrity constraints or both. We have studied all possible syntactic interactions between the literals in the query and the literals in the integrity constraints: positivepositive, negative-negative and negative-positive.

The generalized method, called semantic compilation, enables answers to be found for queries with negative literals in cases for which constructive negation or SLDNF find no answers. We have shown that the answers obtained from a semantically compiled database are correct and, in some cases, complete.

When the generalized semantic compilation is applied to disjunctive databases that are inconsistent with the integrity constraints for some minimal models, a new database is produced whose minimal $r$, dels are all consistent with the integrity constraints if that database exists. In this new database, queries can be answered using any procedure for disjunctive databases without requiring a special mechanism to check the constraints. It remains to be seen how semantic compilation can be applied to disjunctive databases with negation.

\section{Acknowledgments}

Terry Ciaasterland was supported for this work by the Office of Scientific Computing, U.S. Department of Energy, under ('ontract W-31-109-Eng-38. The NSF partially supported Jorge Lobo for this work under The grant \#IRI-92102'20. 


\section{References}

[CGM90] U.S. Chakravarthy, J. Grant, and J. Minker. Logic based approach to semantic query optimization. ACM Transactions on Database Systems, 5(2):162-207, June, 1990.

[Cha85] U. Chakravarthy. Semantic Query Optimization in Deductive Databases. Ph.D. thesis, University of Maryland, Department of Computer Science, College Park, 198.5 .

[Char8] D. Chan. Constructive negation based on the completed databases. In R. A. Kowalski and K. A. Bowen, editors, Proc. $5^{\text {th }}$ International Conference and Symposium on Logic Programming, pages 111-125, Seattle, Washington, MIT Press, August 15-19, 1988.

[Cla78] K. L. Clark. Negation as failure. In H. Gallaire and J. Minker, editors, Logic ard Data Bases, pages 293-322. Plenum Press, New York, 1978.

[FM91] J. A. Fernández and J. Minker. Bottom-up evaluation on disjunctive databases. In K. Furukawa, editor, Proc. International Conference on Logic Programming, pages 660-675, Cambridge, Massachusetts, 1991. MIT Press.

[Ciaa92] T. Ciaasterland. Generating Cooperative Answers in Deductive Databases. Ph.D. thesis, University of Maryland, Department of Computer Science, College Park, 1992. (Technical Report UMIACS-TR-92-107, CS-TR-2968, University of Maryland, October, 1992.)

[CiGL+93] T. Gaasterland, M. Giuli „no, A. Litcher, Y. Liu, and J. Minker. Using integrity constraints to control srarch in knowledge base systems. To appear, Journal of Expert Systems.

[GGMN92] T. Giaasterland, P. Godfrey, J. Minker, and L. Novik. A cooperative answering system. In Andrei Voronkov, editor, Proceedings of the Logic Programming and A utomated Reasoning Conference, pages 101-120, volume 2, Russia, July 1992.

[GMTH] H. Callaire and J. Minker, editors. Logic and Databases. Plenum Press, New York, April 1978.

[Kow79] R. Kowalski. Logic For Problem Solving. Elsevier Science Publishing Co, Inc., Oxford, 1979.

[Llow7] J.W. Lloyd. Foundations of Logic Programming. Springer-Verlag, second edition, 1987.

[M(i90] J. Minker and A. Cial. Producing cooperative answers in deductive databases. In P. Saint-Dizier and S. Szpakowics, editors, Logic and Logic: (irammar for Language Processing. L.S. Horward, Ltd., 1990.

[Przk9b] T. (.. Przymusinski. On constructive negation in logic programming. In E.L. Lusk and R.A. Overbeek, editors, Proc. of the North American Conference of Logic: Programming, ('leveland, Ohio, October 16-20, 1989. Addendum to Proceedings. 
[Rei78] R. Reiter. Deductive question answering on relational databases. In H. Gallaire J. Minker, editor, Logic and Data Bases, pages 149-177. Plenum Press, New York, 1978.

[Ros89] K. A. Ross. A procedural semantics for well founded ne; $;$ ation in logic programs. In Proceedings of the 8th ACM SIGACT-SICMOD-SIGART Symposium on Principle of Database Systems, Philadelphia, Pennsylvania, ACM Press, March, 29-31, 1989. 

